\title{
Percepciones docentes frente a la integración didáctica del deporte escolar
}

\section{Teaching perceptions regarding the didactic integration of school sports}

\author{
http://dx.doi.org/10.17981/cultedusoc.12.2.2021.14
}

Recibido: 31 de mayo de 2020 Aceptado: 16 de noviembre de 2020 Publicado: 1 de julio de 2021

\author{
Victor Hugo Quilindo \\ Universidad del Cauca. Popayán (Colombia) \\ vquilindo@unicauca.edu.co
}

\author{
Didi Hernando Belalcazar-Burbano \\ Universidad del Cauca. Popayán (Colombia) \\ didibelalcazar@unicauca.edu.co
}

Para citar este artículo:

Quilindo, V. y Belalcazar-Burbano, D. (2021). Percepciones docentes frente a la integración didáctica del deporte escolar. Cultura, Educación y Sociedad, 12(2), 241-252. DOI: http://dx.doi.org/10.17981/cultedusoc.12.2.2021.14

\section{Resumen}

El deporte escolar en el contexto de la educación básica primaria es una oportunidad para que la comunidad educativa se involucre en procesos que apuntan a la formación integral de los estudiantes en aspectos que son relevantes en la formación personal y en la vida social. Este articulo tiene como objetivo comprender las percepciones docentes frente a la integración didáctica del deporte escolar en los procesos formativos, específicamente el futbol sala, en una institución educativa de básica primaria en la ciudad de Popayán (Colombia). El estudio se realizó con 8 educadoras vinculadas a la institución desde los grados transición a quinto y para reconocer sus percepciones se utilizó el estudio de casos y técnicas como la observación participante y la entrevista semiestructurada. Emergieron dos categorías relevantes, el deporte como oportunidad para establecer diversas interacciones en el contexto escolar y el deporte como espacio que fortalece la formación integral de los estudiantes. Como conclusión resalta el deporte escolar como agente que promueve procesos de inclusión, interacción, valores y la participación de los diferentes actores educativos y deportivos.

Palabras clave: Deporte; formación; valores; competencia; participación

\section{Abstract}

School sports in the context of basic primary education is an opportunity for the educational community to get involved in processes that aim at the comprehensive training of students in aspects that are relevant in personal training and in social life. This article aims to understand teachers' perceptions regarding the didactic integration of school sports in training processes, specifically futsal, in an elementary school educational institution in the city of Popayán (Colombia). The study was carried out with 8 educators linked to the institution from grades transition to fifth and to recognize their perceptions, case studies and techniques such as participant observation and semistructured interviews were used. Two relevant categories emerged, sport as an opportunity to establish various interactions in the school context and sport as a space that strengthens the integral formation of students. In conclusion, school sports stand out as an agent that promotes processes of inclusion, interaction, values and participation of the different educational and sports actors.

Keywords: Sport; training; values; competition; participation 


\section{INTRODUCCIÓN}

El deporte escolar se asocia a la escuela porque brinda sano esparcimiento a los estudiantes en horario escolar o extraescolar y crea ambientes alternativos fuera de lo cotidiano que permiten la vivencia de situaciones diversas que impactan en el ambiente institucional. Razón por la cual se desarrolló una estrategia centrada en un torneo de futbol sala orientada a la integración interclases que logró la participación de los estudiantes desde diferentes roles, al privilegiar la inclusión y limitar la exclusión por razones de habilidad individual, que suelen ser comunes en la práctica deportiva tradicional.

Se infiere como recurrente que las instituciones educativas privilegien eventos considerados productivos académicamente y sean reacios a integrar las prácticas deportivas por su valor educativo y en ocasiones son los educadores que por falta de tiempo y otras circunstancias académicas no generan propuestas alternativas en las instituciones, por ejemplo, las deportivas que deben "procurar una participación activa y consensuada de todos los agentes sociales que intervienen en dicho proceso deportivo educativo” (Hernández, Martínez y Águila, 2017, p. 41). Frente a lo anterior:

Todo sistema educativo debe asignar el lugar y la importancia debidos a la educación física, la actividad física y el deporte, con miras a establecer un equilibrio y fortalecer los vínculos entre las actividades físicas y otros componentes de la educación (Carta Internacional de la Educación Física, la Actividad Física y el Deporte, 2015, art. 1, inc. 7).

La actividad física, la educación física y el deporte generan condiciones de inclusión de la comunidad educativa y el torneo de integración de fútbol sala abrió un espacio para fortalecer los valores y la interacción de los niños. Es necesario reconocer el potencial formativo de las prácticas deportivas en el contexto escolar que, si bien hacen parte de la cotidianidad de la escuela en la clase de educación física o en las actividades desarrolladas por los estudiantes en tiempos como el recreo, requieren ser organizadas en situaciones incluyentes y participativas no solo para los niños, sino para las docentes.

El torneo de integración de futbol sala en una institución educativa en la ciudad de Popayán (Colombia), desarrollado en los momentos de descanso para potenciar el valor formativo del deporte y para atender situaciones de convivencia institucional y de exclusión, requiere comprender los significados emergentes en las prácticas deportivas al interior del campo y fuera de él, donde se reproducen situaciones de socialización propias de la realidad. "En el proceso de socialización los niños adquieren los roles, expectativas, creencias y valores que la sociedad provee, brindándole al sujeto una sensación de estabilidad frente al orden social en el que está inmerso" (Cruz, 2015, p. 9). Situaciones que merecen ser comprendidas desde los agentes encargados del proceso formativo en la escuela como son las educadoras, razón por la cual el objetivo fue comprender las percepciones docentes ante el torneo de integración de futbol sala en una institución educativa de básica primaria en la ciudad de Popayán (Colombia).

\section{REVISION DE LA LITERATURA}

El deporte como práctica cultural instaurada en las diferentes sociedades y en su origen se supone educativo. Se instala en los sectores académicos a partir de los valores fundamentales, privilegiado la práctica satisfactoria en sí, el goce y la sana competencia como alternativa 
para reorientar las conductas de los niños y jóvenes en todos los contextos sociales. Aspectos como saber ganar y perder, dar el máximo esfuerzo y ser competitivos, son transversales a la práctica deportiva que intrínsecamente promueven valores como la igualdad de oportunidades, la amistad, la actitud responsable durante el juego, dar todo para ganar, esforzarse al máximo y respetar las normas.

Por su parte, el deporte escolar se adscribe al contexto de la educación formal como espacio para el sano esparcimiento, y así crear ambientes alternativos que rompen con la cotidianidad en horario escolar o extraescolar e integrando a la comunidad educativa en prácticas orientadas a la formación integral de los educandos y de los procesos educativos en general. Inicialmente el deporte escolar se dirige a "las prácticas deportivas realizadas por niños y jóvenes, dirigidas por la institución escolar en espacios no lectivos, de participación voluntaria y que tengan un carácter educativo" (Fraile citado en Hoyos, Gutiérrez y Pérez, 2011, p. 20). Por su parte Blázquez (1995), considera que "el deporte escolar remite, en primer lugar y en sentido restringido al tipo de deporte y actividad física que se desarrolla en el marco local de la escuela" (p. 24); para el autor, en sentido amplio representa todo tipo de actividad física realizada al margen de la clase de educación física. Por otra parte:

El deporte escolar lejos está de ser deporte, en el sentido estricto con el que se lo conoce como concepto, es decir, una práctica corporal, competitiva, con reglas que son administradas en general por un árbitro imparcial y regida por algún tipo de asociación o federación, que se da en un espacio y tiempo específico, cuyo objetivo es alcanzar la supremacía en términos de rendimiento de uno o un equipo, por sobre uno o varios oponentes (Hours, 2019, p. 4).

No obstante, la competencia es inherente al deporte en cualquiera de sus formas, lo que genera regularmente exclusión y poca participación por cuanto, "el modelo participativo parece estar relegado a un segundo plano, frente al competitivo, el cual genera un proceso selectivo, discriminatorio y reducido para los escolares" (Hernández et. al., 2017, p. 39). Es necesario reconocer los beneficios de la competición respecto a valores como el compromiso, la responsabilidad, la perseverancia, el sacrificio individual, la honestidad, el respeto a las normas y la búsqueda de objetivos con el trabajo en equipo, por lo cual, "hay que variar la competición hacia una educación deportiva que influya en la inclusión y no en la exclusión" (Hernández et al., 2017, p. 104).

Las actividades deportivas deben aportar a la formación integral de los educandos con la participación de la comunidad educativa (estudiantes, padres de familia, docentes y personal administrativo), en correspondencia con los fines de los procesos formativos en la escuela, "desde esta perspectiva se atribuye así una inmensa responsabilidad a los maestros, profesores, entrenadores, monitores, etc, quienes a través de sus prácticas pueden otorgar una significación distinta en este deporte en beneficio de los niños y jóvenes" (Hoyos et al., 2011, p. 33). Un deporte incluyente, para todos, que vele por el desarrollo integral de los sujetos no solo de las capacidades físicas, sino de las habilidades sociales y psicológicas, en correspondencia con la Carta Internacional de la Educación Física, la Actividad Física y el Deporte (2015) en tanto, "la educación física, la actividad física y el deporte pueden mejorar la salud mental, el bienestar y las capacidades psicológicas al fortalecer la seguridad corporal, la autoestima y la confianza en uno mismo" (art.2; inc. 3).

La Ley 181 (1995) de Colombia, por la cual se dictan disposiciones para el fomento del deporte, la recreación, el aprovechamiento del tiempo libre y la Educación Física y se crea el 
Sistema Nacional del Deporte, define los tipos de deporte, que para el particular se asocia al deporte social, cuyo fin es brindar un espacio de sano esparcimiento, donde los escolares disfruten, se recreen y se integren en un ambiente escolar adecuado y que va encaminado a fortalecer los procesos formativos establecidos por la institución educativa (Ley 181, 1995, título IV, cap. I, art. 16).

Las actividades deportivas en este sentido, deben promover la inclusión, la participación y el fortalecimiento de capacidades individuales y habilidades sociales fundamentado en los valores. En este sentido, el deporte escolar genera beneficios para el crecimiento y desarrollo del niño(a) en aspectos biológicos, psicológicos y sociales, lo que dota al escolar de herramientas para consolidar su autonomía.

Pese a lo anterior, algunas instituciones educativas no dimensionan el potencial educativo del deporte en la escuela y desconocen los múltiples beneficios de las prácticas deportivas en la formación de los estudiantes. Consecuentemente, las prácticas deportivas permiten a la comunidad educativa vivenciar un ambiente alejado de lo cotidiano, de interacción constante con los pares y los profesores, fomentando hábitos saludables y habilidades sociales como la convivencia en pro de mejorar la calidad de vida y atendiendo situaciones problemáticas como la violencia, el sedentarismo, el estrés y la agresividad que afectan el desarrollo físico y social de los escolares, dejando la mirada del deporte competencia donde ganar o no pasa a segundo plano, "El primer objetivo y del deporte curricular y extraescolar es educativo y formativo, la victoria está en función de haber alcanzado el primer objetivo" (Cruz et al. citados en De Bofarull y Cosi, 2014, p. 53).

Además del resultado, en la práctica del deporte no solo es importante el aprendizaje de técnicas o la adquisición de la condición física:

[...] sino que lo realmente, y único educativo son las condiciones en que puedan realizarse esas prácticas que permitan al deportista comprometer y movilizar sus capacidades de tal manera que esa experiencia organice y configure su propio yo, logre su auto-estructuración" (Blázquez, 1995, p. 63).

Por otra parte, "se pretende un desarrollo integral del participante (motriz, afectivo, social, cognitivo) así como una formación en valores, ambos requisitos imprescindibles para plantear una verdadera educación deportiva" (Hernández et al., 2017, p. 40). El deporte escolar significa formación integral y afectación a cada uno de los contextos en los que se desenvuelve el niño, tanto en la escuela como el contexto social en general.

\section{Metodología}

Se empleó un paradigma cualitativo, donde los investigadores "estudian la realidad en su contexto natural, tal y como sucede, intentando sacar sentido de, o interpretar, lo fenómenos de acuerdo con los significados que tienen para las personas implicadas" (Rodríguez-Groba, Eirín-Nemiña y Alonso-Ferreiro, 1999, p. 32). Se recurrió al estudio de casos por permitir "el estudio de la particularidad y de la complejidad de un caso singular, para llegar a comprender su actividad en circunstancias importantes" (Stake, 2013, p. 12).

El estudio de casos permitió comprender los aportes de un torneo de integración de futbol sala en los niños de una institución educativa ubicada en zona rural al occidente de la ciudad de Popayán (Colombia), a partir de diversas situaciones emergentes en los espacios de 
recreo cuya duración es de 30 minutos, con la participación activa de los educandos y de las profesoras durante 2 meses en las actividades deportivas, promotoras de la interacción, el dialogo y la comunicación entre los participantes, en tanto la convivencia institucional suele verse amenazada por la discusión, las diferencias, la exclusión, la rivalidad y la resolución inadecuada de los conflictos. Desde los grados primero a quinto se conformaron los equipos y las educadoras como directoras de grado, cumplieron el rol de dirigir a sus grupos durante los encuentros deportivos, que sin distinción por grado o edad participaron activamente.

Para comprender las percepciones de las educadoras sobre el deporte escolar en las dinámicas institucionales, en espacios alternativos a las aulas de clase que movilizan otro tipo de posibilidades relacionales, se recurrió a la modalidad de estudio de caso intrínseca, en el cual, "el caso viene dado. No nos interesa porque con su estudio aprendamos sobre otros casos o sobre algún problema general, sino porque necesitamos aprender sobre ese caso particular" (Stake, 2013, p. 17). Para la organización y redacción de la información obtenida de las vivencias reales enmarcadas en la participación de las educadoras en el torneo de integración deportiva, se recurrió al estudio de casos en educación, interpretativos, en la intención de analizar las experiencias significativas para las educadoras en medio de las situaciones propias de las prácticas deportivas, su aporte a los estudiantes y al contexto general de la institución. En el énfasis interpretativo:

La interpretación es un proceso cognitivo e intuitivo altamente especializado, y suele requerir una completa inmersión en los datos, la relectura de transcripciones, notas de campo, observaciones y otras formas de datos en el conjunto de éstos, como poemas, viñetas, cameos o relatos (Simons, 2013, p. 167).

El diseño del estudio de caso responde a la propuesta de Simons (2013), que incluye selección del caso específico de interés en el que se desea profundizar, selección de técnicas para recolectar, analizar y comprender el caso, la elección de la muestra dentro del caso para decidir lo que se debe observar y las personas a entrevistar, acceso al contexto especifico del estudio y el análisis interpretativo de la información para comprenderlo.

\section{Participantes}

Se realizó el trabajo en una institución educativa de la ciudad de Popayán (Colombia). Se contó con la participación de 160 estudiantes desde el grado de transición hasta los grados quinto. Se seleccionaron 8 educadoras formadas con título de pregrado de Licenciatura, quienes fueron las informantes clave para el estudio. El proceso estuvo conformado en su totalidad por mujeres, porque todos los grados en la institución están a cargo del sexo femenino.

\section{Procedimiento}

Para el contacto con la institución se solicitó el aval del rector, lo que permitió la comunicación permanente con las educadoras, se informó sobre sus propósitos y se solicitó el consentimiento informado. La identidad de las profesoras se mantiene reservada al utilizar códigos que excluyen nombres propios, con el objeto de respetar la privacidad e intimidad de las educadoras, quienes fueron seleccionadas por estar nombradas en propiedad, contar con 
más de cinco años de experiencia docente en la institución y participar voluntariamente en el proyecto.

\section{Técnicas de recolección de la información}

Las técnicas utilizadas para la recolección de la información fueron la observación, la cual "permite obtener información sobre un fenómeno o acontecimiento tal como este se produce” (Rodríguez-Gómez, Gil-Flores y García-Jimpenez, 1999, p. 150) y la entrevista semiestructurada que pretende, "una verdadera relación de confianza entrevistador entrevistado cuando este último aporta información personal comprometedora para él y cuando tiene suficiente libertad para preguntar al entrevistador" (Rodríguez et al., 1999, p. 172). Para la comprensión de los relatos expuestos en los resultados se realizaron las siguientes codificaciones: E1: corresponde a número de entrevista. FP: corresponde a la secuencia de la Formulación de Pregunta. S: corresponde al Sujeto que responde.

\section{Análisis de la información}

Para el análisis de la información se recurrió a la propuesta de (Rodríguez-Gómez et al., 1999) en reducción de datos para simplificar, resumir y seleccionar la información, diferenciando unidades relevantes y significativas, donde se recurre a la categorización para clasificarlas conceptualmente. Disposición y transformación de los datos para que la información esté ordenada y operativa, de igual modo el cambio en la forma de expresión del lenguaje utilizado. Finalmente se describen las conclusiones como respuesta a los aportes significativos del torneo del futbol sala para los estudiantes y la institución educativa.

\section{Resultados y Discusión}

Aplicado el estudio de casos en la institución educativa alrededor del torneo de integración de futbol sala, emergieron dos categorías de análisis desde los hechos significativos para las educadoras en el contexto educativo, que representan aportes importantes para la convivencia institucional, al visibilizar el deporte como medio de participación e interacción con los pares y educadores.

\section{El deporte. Oportunidad integradora en el escenario escolar}

Las educadoras de la Institución Educativa seleccionada, manifiestan que el deporte escolar y específicamente la realización del torneo de futbol sala permitió la unión entre la comunidad educativa, pues todos esperaban con ansias los encuentros deportivos en horas del recreo, en el cual se sentían incluidos independiente si jugaban o si eran animadores de sus compañeros. "El recreo se constituye como un ambiente deportivo de aprendizaje escolar, donde el espacio y los materiales, contextualizan las posibilidades de desarrollo de las diferentes manifestaciones lúdico-deportivas" (Hernández et al., 2017, p. 44). Para el primer caso, las manifestaciones de alegría y gozo se evidenciaban cuando los niños que jugaban se preparaban para el encuentro y para el segundo caso, sus compañeros de aula se preparaban organizando las porras o barras con el fin de apoyar y alentarlos sin 
importar el marcador final. Por lo cual el recreo, "para él, es un momento único y exclusivo de la escuela, diferente a los demás, en el que se adquieren vivencias personales, desde un marco para la socialización, asociado a un espacio para jugar” (Hernández et al., 2017, p. 47).

Las actividades deportivas permiten que los niños y niñas demuestren diferentes capacidades, por ejemplo, al desempeñarse espontáneamente durante los partidos, situación que no se aprecia en otros espacios de la institución y que además demuestran actitudes que evidencian gozo y alegría durante el desarrollo del torneo, el relato lo muestra, "lo disfrutaron al máximo" (E1: FP4: S1). Además, el deporte en el contexto escolar fomento la práctica de los valores para mejorar la convivencia en la escuela y luego trasladarse a la cotidianidad de los niños, tal y como expresan Escarti y Marín (citados en RodríguezGroba et al., 2017), "el objetivo último del programa es que los alumnos sean individuos eficientes en su entorno social, aprendiendo a ser responsables de sí mismos y de los demás, incorporando las estrategias que les permitan ejercer el control de sus vidas" (p. 88).

Estos espacios deben ser recurrentes en la institución, pues promueven el goce de los educandos y rompen con la monotonía de la cotidianidad de la escuela, ocupando sanamente su tiempo libre, promoviendo la lúdica y el juego. "La acción de jugar, el deporte y la educación son realidades que se complementan, las cuales podemos analizar desde su interacción en diferentes perspectivas, que permiten configurar al deporte como un vehículo educativo" (Hernández et al., 2017, p. 41). Además, ellos toman un rol más participativo dentro de la institución sintiéndose más valorados y aceptados, lo que genera conductas socialmente incluyentes, como se aprecia en el relato «si porque los niños se sintieron valorados, aceptados generando conductas socialmente competentes (E1: FP3: S2).

Desde esta perspectiva el deporte escolar genera impacto positivo en la formación de los niños, debido a que se interioriza principalmente el disfrute, el goce y la satisfacción de ser participe en un compartir con los pares, sin la idea competitiva, de confrontación, de adversidad, de rivalidad y de exclusión típicos en las prácticas deportivas en el escenario escolar. Los profesores juegan un rol importante para recuperar las potencialidades de la práctica deportiva, pues actúan como modelos a imitar por parte de los alumnos/as, por lo que deben resaltar aquellos logros relacionados con una mejora de las habilidades del juego o deporte, los aspectos lúdicos asociados al mismo, el esfuerzo e interés por la mejora en el desempeño de la actividad, y siempre bajo el paraguas de los aspectos éticos, cuestionando el valor de los resultados de la competición frente a los rivales (Rosado, Palma, Mesquita, y Moreno, citados en Rodríguez-Groba et al., 2017, p. 88).

Al sonido de la campana para el descanso, los niños se reúnen en el patio y buscan la mejor ubicación para participar en diferentes roles del encuentro deportivo, utilizan los andenes que rodean la cancha para el inicio del juego, tal y como lo afirma el relato, "dentro del ámbito escolar si, se mantuvo la expectativa de los actores de este proceso que llegara el momento y verlos pendientes a que suene la campana del descanso para reunirse en el patio de recreo de aquel que buscaba su puesto en el andén y que iniciara su partido" (E1: FP1: S4). Así, "en el deporte educativo, es fundamental la participación activa de la persona para que los aprendizajes sean significativos, a partir de una metodología participativa, flexible y contextualizada, donde el participante influye en las experiencias desarrolladas" (Hernández et al., 2017, p. 40). 
Incluso fue visible la participación de los niños más pequeños, la emoción con la que afrontaban los encuentros deportivos, la alegría que irradiaban al tomar el balón y patearlo con la ilusión de ser posiblemente su primera intervención en un escenario de práctica deportiva con sus pares, donde sin importar las normas o un reglamento específico sabían que debían dar lo mejor de sí para sacar a flote sus habilidades y hacer todo lo posible por disfrutar del juego. En esta perspectiva se debe considerar que, "los programas de educación física, actividad física y deporte han de concebirse de tal modo que respondan a las necesidades y características personales de quienes practican esas actividades a lo largo de toda su vida" (Carta Internacional de la Educación Física, la Actividad Física y el Deporte, 2015, art. 4, inc. 1).

Para las educadoras el deporte escolar es un estilo de vida, que incluye la actividad física, la alimentación y la disciplina como bases de un proyecto personal que busca el bienestar para los estudiantes y sin distingos de ninguna clase los incluye a todos por igual, "el deporte es un estilo de vida, es incluir la actividad física, la alimentación, la disciplina y crear un proyecto de vida pensando en el bienestar" (E1:FP6: S7).

En virtud de las formas de relación de los estudiantes con sus pares, las educadoras creen que las posibilidades de las prácticas deportivas se deben trabajar día a día, resaltando por ejemplo el cambio de actitud de los estudiantes conflictivos, quienes además de la indisciplina tenían bajo rendimiento y encontraron en el deporte una motivación para cambiar sus actitudes, "la apuesta a que cambiaran de actitud sobre todo los estudiantes con conflictos disciplinarios, de bajo rendimiento académico, total conformidad con la perdida de año escolar, a las cuales personalmente les hice el reto que si mejoraban podían estar en los equipos que de lo contrario hablaría con el profesor de educación física al respecto y que de seguro estaba de acuerdo (E1: FP8: S3), “... el sistema educativo y aquellos espacios de gran significación para el alumnado, como es la práctica deportiva, han de contribuir en la adquisición de valores sólidos, tanto en los aspectos más individuales como en los convivenciales" (Hernández et al., 2017, p. 57).

El deporte escolar debe promover los valores por el alto beneficio educativo que tiene su práctica, "Uno de los principios, tal y como venimos remarcando, es la importancia de trabajar por la tolerancia y el respeto, eliminando conductas discriminatorias que tienen lugar en el deporte base" (Rodríguez-Groba et. al., 2017, p. 102). Gracias a estos eventos deportivos, la institución educativa se muestra incluyente, al fortalecer procesos formativos integradores, además son puente motivacional para que los estudiantes asistieran a clases con dinamismo y que la comunidad evidenciara otras posibilidades para la enseñanza aprendizaje en la institución. Al respecto, "nos mostramos como una escuela incluyente que forma personas integralmente, motivó a la asistencia a clases y eso hace que la comunidad vea que se desarrollan procesos diferentes de aprendizaje (E1:FP10: S7). Es relevante fomentar actividades que reúnan a la comunidad educativa en propósitos comunes, que aportan a los procesos de enseñanza aprendizaje en la escuela, de esa manera los educandos encontraran otros motivos para disfrutar de ese escenario fundamental en su formación como ciudadanos, pues las prácticas deportivas responden a sus necesidades, intereses, motivaciones en ambientes agradables que favorecen el aprendizaje y la interacción con sus pares. 


\section{El deporte escolar formativo y competitivo}

La percepción sobre el desarrollo del torneo tuvo dos características. Por un lado, el carácter competitivo propio de las prácticas deportivas y por el otro, el formativo necesario en todo tipo de prácticas, quedando demostrado por ejemplo en el deseo de los estudiantes por ganar en cada uno de los encuentros deportivos con el apoyo de sus compañeros y también la aceptación responsable y respetuosa cuando el resultado no se daba, aceptándolo y sobreponiendo la diversión como objetivo principal de la actividad. El deporte escolar según las percepciones de las educadoras, aporta a la formación integral de los educandos en el contexto institucional, “y así se convierte en un proyecto de extrema necesidad para la escuela en un grado de importancia inconmensurable en la formación integral de niños y jóvenes en la institución" (Paiva, Carvalho \& Batista, 2020, p. 14).

La competencia es inherente al deporte, en ella los participantes demuestran sus habilidades individuales y las ponen al servicio del colectivo de manera autónoma, en función de la naturaleza del deporte específico. Sin embargo, también debe aportar a la formación integral no solo para quienes están en campo, sino para quienes se involucran desde otras responsabilidades, motivo por el cual los educadores deben promover actividades deportivas en la escuela, principalmente quienes se desempeñan en el área "Por lo tanto, los profesores de educación física deben fomentar que los estudiantes realicen AF de forma autónoma, lo que con suerte ellos luego se aplicará a la actividad física durante su tiempo libre tiempo sin la ayuda de profesores" (Casado-Robles, Mayorga-Vega, Guijarro-Romero \& Viciana, 2020, p. 10).

Lo formativo se apreció cuando la convivencia escolar se vio mejorada por la fluidez en la interacción entre estudiantes y profesores, logrando actitudes como aprender a compartir experiencias con los compañeros del propio grado o de otro grupo o comprender el valor de ganar o perder en un encuentro deportivo sin peleas o discusiones con sus compañeros por el marcador de los partidos o por el número de goles, "también aprendieron a ganar y a perder las competencias sin ningún rencor ni pelea entre sus compañeros (E1: FP9: S2). Se resalta la importancia de la competencia y la socialización en el deporte escolar, así como otros valores expuestos por Paiva et al. (2020) donde evidencian el impacto de las propuestas para los niños, "y demuestran que el deporte escolar valora la integración, la disciplina, el crecimiento personal y comunicación que establecen en estas actividades" (p. 13).

Al iniciar el torneo, la intención competitiva embargaba a muchos niños, pero con el transcurso del mismo, las actitudes de alegría, disfrute, goce, interacción, compartir y otra serie de valores pasaron a ser fundamentales, "creo que en un principio evidenciaron comportamientos competitivos, pero a medida que fue avanzando el proceso se volvió formativo (E1: FP3: S3). "Por lo tanto, la competición en si no es negativa, sino su mala interpretación y aplicación en la enseñanza" (Giménez, 2000, p. 106). Las características formativas impactaron en la comunidad educativa, contribuyendo en la formación integral de los participantes, pues en las diferentes situaciones emergentes en los encuentros deportivos pasaron por momentos de dialogo, discusión amistosa y de liderazgo, percibido en situaciones que promovieran el bien para el equipo, demostrado no en la rivalidad con el otro, sino dando todo de sí para lograr el mejor desempeño al interior del campo, lo que 
finalmente culminó en mejores espacios de convivencia dentro de la institución educativa. La competencia en el deporte escolar no es formativa por sí misma, se requiere de acciones planeadas, cargadas de ese potencial formativo.

Si queremos convertir la competición en un elemento formativo es necesario que vaya acompañada de actuaciones concretas que eviten que ese potencial formativo se quede en la teoría, consiguiendo que actitudes y valores positivos estén pues en la práctica deportiva cotidiana. (Monjas, Ponce y Gea, 2015, p. 6).

El deporte escolar brindó un espacio lúdico-recreativo que fomentó valores, creando lazos de amistad, en pro de aprendizajes significativos en la vida cotidiana de los estudiantes. También buscó fomentar hábitos y estilos de vida saludables para prevenir problemáticas recurrentes en la actualidad como el consumo de sustancias psicoactivas o la delincuencia, "las finalidades educativas y saludables son, actualmente las que diferentes especialistas en este ámbito concuerdan en otorgar a las actividades físicas y deportivas en edad escolar" (Lleixa, González, Monguillot, Daza y Braz, 2015, p. 28). Al respecto estas actividades, "fomentan y promueven el bienestar del niño o niña para no caer en la drogadicción, la delincuencia social, etc. Hace parte de una vida saludable. Sensibiliza y anima la participación. Hace perder temores y miedos. Despierta interés y desarrolla habilidades" (E1: FP6: S4). El deporte escolar fortalece las habilidades individuales y sociales de los estudiantes, relacionadas con la consecución de un resultado, pero sobretodo promueve la relación con el otro, el fortalecimiento de los valores y beneficia la convivencia en el contexto institucional, siempre que su práctica contenga el valor educativo.

El torneo de futbol sala permitió la expresión de habilidades motrices no posibles en otros espacios escolares, de igual modo el fomento de valores para la formación integral. Esta percepción acerca del deporte se asocia a una motivación intrínseca de los estudiantes articulada a los valores y el compromiso, "los escolares con más deportividad serán los que muestren más inclinaciones cooperativas (task-oriented) y más motivación intrínseca" (De Bofarull y Cosi, 2014, p. 53), reafirmado en el relato "si soy buen deportista puedo ser mejor ser humano colaborador, receptivo, solidario, manejar emociones” (E1: FP6: S3).

El torneo de futbol sala cambio la manera de asumir la competencia deportiva, al ser respetuosa y con decisiones responsables durante los encuentros, "este torneo o evento genero la sensación de cambio en su forma de competir con otros compañeros de otros grados que deben realizarse con respeto y responsabilidad (E1: FP10: S2). A propósito, "lo que se requiere no son solo manifestaciones de las virtudes sino comportamientos genuinamente comprometidas con las formas de conducta que se exige" (Blázquez, 1995, p. 34).

En el deporte escolar el carácter competitivo adquiere valor educativo cuando se construyen situaciones distantes de la exclusión, la agresividad y ganar a cualquier costo, pues "son las condiciones en las que se desarrollan las prácticas deportivas - entre ellas las competiciones - las que configuran el carácter educativo o no del deporte" (Manzino y Rodríguez, 2016, p. 7), lo que aporta a la formación integral en aspectos como la adquisición de valores, la resolución pacífica de conflictos, la participación activa, la autonomía, el liderazgo, aprender de la victoria y la derrota, resaltar todo tipo de habilidades, mejorar la percepción de sí mismo, adquirir responsabilidades académicas y ser personajes activos en la construcción de los propósitos formativos de la escuela. 


\section{Conclusiones}

Las actividades deportivas con propósitos de inclusión y participación en el contexto escolar, posibilitan la interacción de los niños con sus pares y con los educadores en las diversas situaciones emergentes al interior del campo de juego y las circunstancias alrededor del mismo, en las cuales todos los agentes son relevantes, independiente de rol asumido durante el desarrollo de las actividades deportivas que logran aportes significativos para el reconocimiento, así como los procesos de socialización en la escuela.

Los eventos deportivos escolares con prioridad en el disfrute y el goce, provocan rupturas en las dinámicas competitivas tradicionales que privilegian la exclusión o el resultado a cualquier costo y, por el contrario, aportan en la formación integral con el fortalecimiento de los valores, el desarrollo de habilidades sociales, el respeto a las normas e incremento del bienestar en la convivencia, que son aspectos inherentes a la competencia en el deporte, en tanto en su planificación y desarrollo existan intencionalidades formativas.

\section{REFERENCIAS}

Blázquez, D. (1995). A modo de introducción. En, D. Blázquez, (Ed.), La iniciación deportiva y el deporte escolar (pp. 18-46). Barcelona: INDE.

Casado-Robles, C., Mayorga-Vega, D., Guijarro-Romero, S. \& Viciana, J. (2020). Sport education-based irregular teaching unit and students' physical activity during school recess. Journal of Educational Research, 113(4), 262-274. https://doi.org/10.1080/002 20671.2020.1806014

Cruz, M. (2015). La Niñez en la escuela: Una historia de poder, control y desarrollo. Cultura Educación y Sociedad, 6(1), 127-140. Disponible en https://revistascientificas.cuc. edu.co/culturaeducacionysociedad/article/view/759

De Bofarull, I y Cusi, M. (2004). Deportividad en el deporte escolar y extracurricular. Apunts: educación física y deporte, (116), 52-59. http://dx.doi.org/10.5672/ apunts.2014-0983.es

Giménez, J. (2000). Fundamentos básicos de la iniciación deportiva en la escuela. Sevilla: Wanceulen.

Hernández, A. I., Martínez, L. F. y Águila, C. (Coord.) (2017). El deporte escolar en la sociedad contemporánea. Almería: Editorial Universidad de Almería.

Hoyos, L. A., Gutiérrez, C. y Pérez, Á. (2011). Deporte Escolar. Fundamentación Teórica. Armenia: Kinesis.

Hours, G. (2019). El Deporte Escolar Persigue La Institucionalización. / School Sports Pursue Institutionalization. Materiales para la Historia del Deporte, (18), 41-55. Recuperado de http://www.memoria.fahce.unlp.edu.ar/art_revistas/pr.9268/pr.9268.pdf

Lleixa, T., González, C., Monguillot, M., Daza, G. y Braz, M. (2015). Indicadores de calidad para los centros escolares promotores de actividad física y deportiva. Apunts: educación física y deporte, (120), 27-35. http://dx.doi.org/10.5672/apunts.2014-0983. es.(2015/2).120.04 
Manzino, C. y Rodríguez, V. (2016). Sí al deporte en la escuela, pero... ¿y las competiciones deportivas escolares? Revista Universitaria de la Educación Física y el Deporte, 9(9), 15-23. Disponible en http://www.revistasiuacj.edu.uy/index.php/rev1/article/view/99

Monjas, R., Ponce, A. y Gea, J. (2015). La transmisión de valores a través del deporte. Deporte escolar y deporte federado: relaciones, puentes y posibles trasferencias. RETOS. Nuevas Tendencias en Educación Física, Deporte y Recreación, 28, 276-284. https:// doi.org/10.47197/retos.v0i28.35650

Organización de las Naciones Unidas para la Educación, la Ciencia y la Cultura. (17 de noviembre de 2015). Carta Internacional de la Educación Física, la actividad física y el deporte y el Deporte. [Online]. Disponible en http://portal.unesco.org/es/ev.phpURL_ID=13150\&URL_DO=DO_TOPIC\&URL_SECTION=201.html

Paiva, L., Carvalho, M. J. \& Batista, P. (2020). Dinâmica e Valor do Desporto Escolar no contexto da Gestão da Escola La Salle Manaust. Revista Intercontinental de Gestão Desportiva, 10(1), 1-19. Disponível em http://www.revista.universo.edu.br/index.php?journal=gestaoesportiva\&page=article\&op=viewArticle\&path $\% 5 B \% 5 \mathrm{D}=8011$

República de Colombia. Congreso de la República. (18 de enero de 1995). Por el cual se dictan disposiciones para el fomento del deporte, la recreación, el aprovechamiento del tiempo libre y la Educación Física y se crea el Sistema Nacional del Deporte. [Ley 181]. Recuperado de https://www.mineducacion.gov.co/1621/articles-85919_archivo_pdf.pdf

Rodríguez-Gómez, G., Gil-Flores, J y García-Jimpenez, E. (1999). Metodología de la Investigación Cualitativa. Málaga: Aljibe.

Rodríguez-Groba, A., Eirín-Nemiña, R. y Alonso-Ferreiro, A. (2017). Materiales y recursos didácticos contra la discriminación y la exclusión en el deporte en edad escolar: el proyecto "IRIS". Educatio Siglo XXI, 35(3), 85-104. https:/doi.org/10.6018/j/308911

Simons, H. (2013). El estudio de caso: teoría y práctica. Madrid: Morata.

Stake, R. E. (2013). Investigación con estudio de casos. Madrid: Morata.

Víctor Hugo Quilindo es Licenciado en Educación Básica con énfasis en educación física Recreación y deportes, actual profesor del departamento de Educación Física, Recreación y Deporte de la Universidad del Cauca. Realizó sus estudios de especialización en la Institución Universitaria Escuela Nacional del Deporte (Santiago de Cali, Valle del Cauca) y de Maestría en las Universidad de Manizales (Manizales, Caldas). En su transcurso profesional cumple labores relacionadas con la docencia, la investigación y la interacción social en programas de pregrado y posgrado. Miembro activo del grupo de investigación Konmoción de la Universidad del Cauca, Colombia. Su producción intelectual se ubica en los campos de la educación física y el deporte. https://orcid.org/0000-0001-7106-1431

Didi Hernando Belalcazar Burbano es Licenciado en Educación Básica con énfasis en educación física Recreación y deportes. Culmine estudio de especialización en entrenamiento deportivo en la Universidad del Cauca. En su transcurso profesional cumple labores relacionadas con la docencia, la investigación y la interacción social a través de la práctica deportiva del futbol sala. https://orcid.org/0000-0002-5044-4860 